\title{
Reportes Integrados voluntarios, ¿es información integrada?
}

\section{Voluntary Integrated Reports, Is it integrated information?}

\author{
Felipe Zúñiga P. ${ }^{1}$, Roxana Pincheira L. ${ }^{2}$, \\ María Aguilar ${ }^{3}$, Gustavo Maragaño ${ }^{4}$
}

\begin{abstract}
RESUMEN
En los últimos 40 años el número de reportes independientes asociados a materias medioambientales, sociales y de gobiernos corporativos se ha incrementado considerablemente. A fines del 2013 se publica el marco conceptual de Reportes Integrados (RI) que integra aspectos financieros y no financieros en una sola narrativa mostrando cómo la organización crea valor en el tiempo. Mediante el desarrollo de un índex basado en el Marco Conceptual Internacional de los Reportes Integrados, este artículo investiga los factores que motivan la calidad de las divulgaciones corporativas de los RI presentados al cierre contable 2018 en el mercado chileno. El índice permite evaluar qué se está divulgando como información integrada y qué impulsa la variación en la calidad de estos reportes. Mediante el análisis de todos los RI divulgados, los resultados sugieren un alto grado de discrecionalidad en la información presentada. Existe información incompleta e inexacta, lo que se contrapone a la esencia de los RI. Se concluye que el proceso aún no está institucionalizado y que, contrario a las expectativas, no ha estimulado un nuevo mecanismo integrado de divulgación en Chile. Los resultados obtenidos en este estudio no son consistentes con lo que, en teoría, son los RI. Se debe tener cuidado al evaluar la información corporativa a través de un informe denominado "integrado" cuando no hay un análisis en profundidad del contenido mínimo requerido de acuerdo con los estándares internacionales.
\end{abstract}

Palabras clave: índex de calidad, información voluntaria, reportes integrados, IIRC.

Recepción: 16/04/2021. Aprobación: 25/10/2021.

\footnotetext{
1 Universidad Austral de Chile, Facultad de Ciencias Económicas y Administrativas, Valdivia, Chile, e-mail, felipe. zuniga@uach.cl

2 Universidad Austral de Chile, Facultad de Ciencias Económicas y Administrativas, Valdivia, Chile, e-mail, roxana. pincheira@uach.cl

3 Universidad Austral de Chile, Facultad de Ciencias Económicas y Administrativas, Valdivia, Chile, e-mail, mariafda.92@ icloud.com

4 Universidad Austral de Chile, Facultad de Ciencias Económicas y Administrativas, Valdivia, Chile, e-mail, tavo96@ gmail.com
} 


\begin{abstract}
In the last 40 years, the number of independent reports associated with environmental, social and corporate governance issues has increased considerably. At the end of 2013, the Integrated Reporting (IR) conceptual framework was published, which integrates financial and non-financial aspects into a single narrative showing how the organization creates value over time. Through the development of an index based on the International Integrated Reporting Conceptual Framework, this article investigates the factors that motivate the quality of corporate disclosures of integrated reports (IR) in 2018. The index assesses what is being disclosed as integrated information, and what drives the variation in the quality of these reports. Through the analysis of all reports informed. The results suggest a high degree of discretionary disclosures in the information presented. There is incomplete and inaccurate information, which is contrary to the essence of the IRs. It is concluded that the process is not yet institutionalized and, contrary to expectations, it has not stimulated a new integrated disclosure mechanism in Chile. The results obtained in this study are not consistent with what, in theory, an IR is. Care must be taken when evaluating corporate information through a so-called "integrated" report when there is no in-depth analysis of the minimum content required on an IR.
\end{abstract}

Keywords: quality index, voluntary information, integrated reporting, IIRC.

\title{
INTRODUCCIÓN
}

Las necesidades de los usuarios de la información empresarial han ido cambiando a través del tiempo. Se ha argumentado que la información no financiera afecta el valor de los activos tangibles y puede estar vinculada a otros activos intangibles como el capital intelectual, la reputación de la empresa, entre otros. Estudios que vinculan componentes del S\&P 500 muestran que el valor de las compañías atribuibles a activos tangibles ha decrecido notoriamente. En los años 70, el valor de mercado de las firmas atribuido a este tipo de activos era superior al 80\%, en el año 2015, el valor promedio de las corporaciones representado por activos físicos y financieros fue inferior al 20\% (Ocean Tomo, 2015). Coincidencia o no, el número de reportes independiente de responsabilidad social corporativa se incrementó en la década de los noventa (Dhaliwal, Radhakrishnan, Tsang, y Yang, 2012). Si bien existe variada evidencia sobre el beneficio de reportar información voluntaria a través de reportes independientes (Dhaliwal, Li, Tsang, y Yang, 2011; Dhaliwal et al., 2012; Diamond y Verrecchia, 1991; Lu y Chueh, 2015; Qiu, Shaukat, y Tharyan, 2016; Zúñiga, Pincheira, Walker, y Turner, 2020), en ocasiones estos reportes han sido criticados por ser difíciles de leer, no enfocados en aspectos materiales, incompletos y desconectados de la estrategia del negocio (Guidry y Patten, 2010; Stent y Dowler, 2015). Motivados por las debilidades de estos reportes corporativos a fines del 2013, el consejo internacional de RI (International Integrated Reporting Council - IIRC por su sigla en inglés) publicó el Marco Conceptual Internacional de RI (IIRC, 2013b) con el objetivo de guiar la preparación y presentación de estos reportes corporativos. Su objetivo, en términos generales, es integrar la información financiera y no financiera en un informe y mostrar cómo las compañías están creando valor en el corto, mediano, y largo plazo a través del uso de capitales financieros y no financieros. Los capitales identificados por el Consejo Internacional son financieros, manufactureros, intelectuales, humamos, sociales y el capital natu- 
ral (IIRC, 2013a). El objetivo principal de los RI es mejorar la calidad de la información disponible para los proveedores de capital financiero, para los accionistas o para los inversores mediante la comunicación de información más amplia y relevante que pueda ayudar en las decisiones sobre la asignación efectiva de capital (IIRC, 2013b). Investigaciones indican que los inversores utilizan los RI para gestionar el riesgo de inversión, evaluar la dinámica de la industria y el entorno regulatorio, y evaluar la información prospectiva de una empresa (IIRC, 2015). En teoría, los RI proporcionan información prospectiva sobre cómo la empresa crea valor a corto, mediano y largo plazo al informar una amplia gama de factores no sólo financieros, sino intelectuales, humanos, sociales y naturales que impactan en el proceso de creación de valor.

Debido al corto período en el que el Marco Internacional de RI ha estado disponible, y considerando que la emisión de estos informes es voluntaria en la mayoría de los mercados, la evidencia sobre los incentivos para divulgar estos reportes se encuentra en una etapa muy temprana. Principalmente, los esfuerzos se han enfocado en estudiar los procesos institucionales de adopción de RI (Brown y Dillard, 2014; Higgins, Stubbs, y Love, 2014; Stubbs y Higgins, 2014; van Bommel, 2014) y otros estudios han determinado los impactos económicos que tiene la divulgación estos reportes corporativos (Barth, Cahan, Chen, y Venter, 2017; Zhou, Simnett, y Green, 2017; Zúñiga, Pincheira, Walker, et al., 2020). Por ejemplo, estudios analizan el proceso de transición de los primeros usuarios de RI en Australia (Brown y Dillard, 2014; Higgins et al., 2014; Stubbs y Higgins, 2014; van Bommel, 2014). Se concluye que el proceso aún no está institucionalizado y que, contrario a las expectativas, no ha estimulado un nuevo mecanismo de divulgación. Defensores de los RI sugieren que el divulgar información integrada reduce la asimetría de la información. Se argumenta que los RI mitigan en cierta medida la brecha entre los informes de sustentabilidad y los reportes financieros, y que sus beneficios se encuentran condicionados a la calidad de la información. Así mismo, la creciente literatura sobre RI ha investigado si la divulgación de información integrada ayuda a los analistas financieros a predecir sus pronósticos. Autores demuestran que la calidad de los RI se encuentra asociada a una mayor precisión en el pronóstico de las ganancias por acción (Zúñiga, Pincheira, Walker, et al., 2020). Por tanto, los RI deberían beneficiar a los proveedores de capital financiero (inversores) y sus representantes (analistas financieros) al destacar la relevancia de valor de las actividades de sostenibilidad en los mercados de capitales. Sin RI, las divulgaciones de sostenibilidad no tienen relevancia directa en el mercado para los proveedores de capital (Bernardi y Stark, 2016). El objetivo de los RI no es sólo integrar la información financiera y no financiera en un solo reporte, sino integrar el modelo de negocio y determinar cómo los capitales crean valor en el tiempo. El público objetivo principal de los RI son los proveedores de capital, lo que implica una alta personalización de la industria (ya que el RI se basa en principios) y un bajo nivel de auditoría porque la información no financiera es más difícil de asegurar que la información financiera.

Ha existido acuerdo en que los beneficios de los RI están asociados a la calidad información presentada. Esto se ha visto reflejado a través de en un menor costo de capital (Zhou et al., 2017), mayor liquidez de mercado (Barth et al., 2017; Zúñiga, Pincheira, Walker, et al., 2020) y una mayor precisión y menor dispersión en los analistas financieros al pronosticar las ganancias por acción (Bernardi y Stark, 2016; Zhou et al., 2017; Zúñiga, Pincheira, Walker, et al., 2020). La evidencia es robusta en el análisis de la calidad de los reportes; sin embargo, todos estos estudios se han realizado en el mercado sudafricano donde la divulgación de los RI es obligatoria por normativa. 
Analizando la divulgación voluntaria en Chile, estudios argumentan que la emisión de reportes de sustentabilidad tiene un efecto positivo en el mercado. La evidencia sugiere que aquellas empresas que presentan reportes externos de sustentabilidad tienen mejores indicadores de liquidez (utilizando como proxy Bid-ask spread), y cuyo efecto se ve incrementado posterior a la aplicación de la normativa N³85 (Zúñiga, Pincheira, Aguilar, y Silva, 2020). Los autores argumentan que sólo el $34 \%$ de la muestra analizada presentó reportes externos de sustentabilidad para el periodo 2013-2017.

Cuando se analizan los efectos económicos de la publicación de RIs, la evidencia es mixta. Por un lado se ha demostrado que, posterior a la publicación del Marco Conceptual en el 2013, la divulgación de los RI afecta positivamente la liquidez organizacional de las empresas chilenas, pero por otro lado, no se encuentra evidencia de que estos reportes tengan un efecto en la precisión de los pronósticos de las ganancias por acción realizado por los analistas financieros (Zúñiga, Pincheira, Dimter, y Quinchel, 2021). El porcentaje de compañías que deciden presentar la información integrada da no es alentador. En promedio sólo el 10,4\% de las empresas que están o han estado en el Índice de Precios Selectivos de Acciones (IPSA), han divulgado su información financiera y no financiera de manera integrada (Zúñiga et al., 2021), a pesar de que la Norma de Carácter General 385 (SVS, 2015) establece, entre otras cosas, el desarrollo de reportes corporativos basados en los Reporting Initiative (GRI) o los estándar utilizados para la preparación y presentación de RI. Lo anterior demuestra que, en Chile, el proceso de divulgación corporativa de materias medioambientales, sociales y de gobiernos corporativos aún no se encuentra institucionalizado.

Este estudio busca a través de la preparación y evaluación de un índex de calidad, basado en el Marco Conceptual Internacional de RI, determinar cuál es la calidad de la información presentada en el mercado chileno y si los reportes, que se denominan integrados, cumplen con los contenidos mínimos establecidos en el Marco Conceptual

El resto de este artículo está organizado de la siguiente manera. Procedimientos de selección de muestras y desarrollo del índice se describen en Sección 2. Las secciones 3 y 4 resumen los resultados y conclusiones respectivamente.

\section{METODOLOGÍA MATERIALES Y MÉTODOS}

Este estudio utiliza la base de datos Datastream de Thomson Reuters para determinar las empresas que están o han estado incluidas dentro del Índice de Precio Selectivo de Acciones (IPSA) de la Bolsa de Comercio de Santiago. Datastream cubre las 108 empresas más grandes del mercado chileno que son reguladas por la Comisión para el Mercado Financiero (CMF). Se analizaron todas las compañías que al cierre contable de 2018 presentaron RI. Se estudian los RI mediante un índice de calidad preparado en base a los principios establecidos en el Marco Conceptual Internacional de RI (IIRC, 2013b). El índex no busca evaluar un tipo específico de divulgación (por ejemplo, ambiental o social), más bien, examina la divulgación de la empresa en su conjunto en términos de su contenido y la forma en que se presenta la información. El índice se basa en los elementos mínimos que debe tener un RI de acuerdo con el Marco Conceptual Internacional. Los elementos de contenido están fundamentalmente vinculados entre sí y no son mutuamen- 
te excluyentes. Es importante mencionar que el orden de los elementos que muestra el Marco Conceptual, y que son la base de la construcción del índex, no es la única forma en que podrían secuenciarse; en consecuencia, los elementos no están destinados a servir como una estructura estándar para un informe integrado. Más bien, la información en un informe integrado se presenta de una manera que hace aparentes las conexiones entre los elementos que contiene el RI. Por lo tanto, el contenido del informe integrado dependerá de las circunstancias individuales de la organización. En este sentido, el Marco Conceptual expresa lo elementos en forma de preguntas y no como listas de verificación de divulgaciones específicas. En consecuencia, se debe ejercer un juicio al aplicar los principios básicos para determinar qué información se informa, cómo se informa y cómo se integra en la unidad de negocio como un todo.

El índex cuenta con seis ejes principales (IIRC, 2013b): (1) Estructura integrada de informes y comunicación efectiva, (2) Estrategia de organización, (3) Materialidad, (4) Ambiente externo, (5) Gobernabilidad, (6) Rendimiento y su perspectiva. Por cada uno de los ejes se generan subfactores. Cada sub-factor recibe una puntuación en una escala de tres puntos. Siguiendo a Herbohn, Walker, y Loo (2014) se asigna un valor de 0 a las empresas donde el elemento relevante no se divulga o no cumple con las pautas aplicables del Marco Conceptual Internacional. Se da un valor de 1 cuando hay una discusión general del ítem especificado, y se otorga un valor de 2 cuando hay una revelación específica sobre el ítem evaluado. El índice cuenta con 33 sub-factores; por lo tanto, el puntaje máximo que podría obtener un reporte es de 66 puntos. El objetivo, es comprender qué tipos de empresas están presentando RI, qué están divulgando, y qué impulsa la variación en la calidad de estos reportes.

Se tomó como muestra inicial la base de datos Datastream de Thomson Reuters que cubre las 108 empresas chilenas más grandes que están o han estado en el Índice de Precio Selectivo de Acciones (IPSA). De la muestra inicial, tomada al cierre contable 2018, el 18,5\% de los datos analizados (20 sociedades anónimas) presentaron su información a través de RI.

\section{Tabla 1}

Criterios del índice de calidad de divulgación de IR.

FACTOR 1. Estructura integrada de informes y comunicación efectiva (puntaje total 0-12)

SUB-FACTOR

1. Sigue los principios básicos de los RI

\section{EXPLICACIÓN}

Los RI se preparan en respuesta a los requisitos estabilizados en el IIRF como un informe independiente o se incluye como una comunicación perceptible e identificable, teniendo una declaración que haga referencia al Marco Conceptual de RI (Principios y elementos de contenido) como base de preparación. (0-2)

2. Conectividad y valor de interdependencia

3. Estructura del informe
Debe proporcionar conectividad e interdependencia sobre los factores que afectan la capacidad de crear valor. (0-2).

El RI proporciona una estructura de informe efectiva, lógica compresible en una secuencia de capítulos, evitando informes largos y estáticos. (0-2) 
4. Conectividad de la Existe conectividad (interrelación y dependencias) entre la información. información proporcionada por la empresa que afecta la capacidad de la organización para crear valor con el tiempo. Esta conectividad se aprecia entre: los elementos del informe; la información pasada, presente y futura; los capitales; la información financiera y no financiera; la información cuantitativa y cualitativa; el gobierno y todos los canales de comunicación de la compañía. (0-2)

5. Optimizar la Incluye referencias cruzadas internas, según corresponda, para legibilidad limitar repetición, evitando revelaciones altamente genéricas que no son específicas de la organización (repetitivo). Además, expresa el concepto claramente y en la menor cantidad de palabras posible, incluyendo gráficos, imágenes y todas las explicaciones necesarias que ayudan a los lectores a navegar por el documento. (0-2)

6. Dimensión del Incluye una descripción clara sobre la dimensión del tiempo futuro tiempo futuro (corto, mediano y largo plazo) considerado en la preparación y presentación del RI. (0-2)

FACTOR 2. Estrategia de organización (puntaje bruto 0-20)

SUB-FACTOR

1. Misión y visión

\section{EXPLICACIÓN}

Proporciona una misión y visión clara de la organización. El RI debe mostrar claramente cuál es la estrategia de la empresa y cómo se relaciona con el proceso de creación de valor a corto, mediano y largo plazo. $(0-2)$

2. Actividades comerciales y mercado (s) en funcionamiento.

3. Riesgos internos y oportunidades

Describe las principales actividades comerciales y los mercados donde opera la empresa. (0-2)

Da una imagen representativa de los riesgos y oportunidades que enfrenta internamente la empresa; cómo afectará la capacidad de la empresa para crear valor a corto, mediano y largo plazo; y cómo la empresa se ocupa de ellos. (0-2)

4. Cultura, ética y valores.

Describe la organización: cultura, ética y valores, propiedad y estructura operativa, e información cuantitativa clave (por ejemplo, el número de empleados, los ingresos y el número de países en los que opera la organización). (0-2)

5. Capitales utilizados
Describe individualmente los recursos (capitales utilizados por la organización para crear valor (incluida la compensación)).

Estos se determinan por su efecto sobre la capacidad de la organización para crear valor a lo largo del tiempo, en lugar de si son o no propiedad de la empresa (si éstos no son fácilmente cuantificables, cualitativos revelados se hacen para explicar sus efectos). (0-2) 
6. Comprensión e interdependencias entre capitales

7. Información de las perspectivas divulgada
Promueve la comprensión y las interdependencias de cada capital, a corto, mediano y largo plazo. (0-2)

La divulgación de información implica diferentes perspectivas, que incluyen: aspectos internos, externos, positivos y negativos respaldados en una relación constante con proveedores de capital financiero y partes interesadas. (0-2)

Describe el modelo de negocio clave que incluye insumos, actividades comerciales, salidas y resultados. (0-2)

i) Entradas. Un RI muestra los aportes materiales más importantes de los que depende la organización. Estas revelaciones de capitales deben tener una dependencia importante para crear valor con el tiempo.

ii) Actividad de negocios. Un RI describe actividades comerciales clave; por ejemplo: cómo la organización se diferencia en el mercado, hasta qué punto el modelo de negocio depende de la generación de ingresos después del punto de venta inicial, cómo innova la organización y cómo se ha diseñado el modelo de negocio para adaptarse al cambio.

iii) Salidas. Un RI identifica cuáles son los productos y servicios clave.

iv) Resultados. Tanto los resultados internos (por ejemplo, la moral de los empleados, la reputación de la organización, los ingresos y los flujos de efectivo) como los resultados externos (por ejemplo, satisfacción del cliente, pagos de impuestos, lealtad a la marca y efectos sociales y ambientales). Tanto los resultados positivos (es decir, aquellos que resultan en un aumento neto de los capitales y, por lo tanto, crean valor) y los resultados negativos (es decir, aquellos que resultan en una disminución neta de los capitales y, por lo tanto, disminuyen el valor)

9. Cómo se manifiesta el valor creado

El valor creado por la empresa se manifiesta a través de: el valor creado por la empresa y otros (es decir, las partes interesadas y la sociedad en general).

10. Valor de efectos creado
Explica cómo la creación de valor de la empresa afecta a los grupos de interés y proveedores de capitales financieros (o accionistas) a lo largo del tiempo.

FACTOR 3. La Materialidad (Puntaje bruto 0-8)

\section{SUB-FACTOR}

1. Existencia de un proceso

2. La forma en que se han determinado y cuantificado

\section{EXPLICACIÓN}

Existencia de un proceso que determine los aspectos materiales que son considerado en el RI; (0-2)

La forma en que se han determinado y cuantificado. Evalúa la importancia de cosas materiales. En base a la magnitud de sus efectos (riesgos oportunidades y resultados) y la probabilidad de ocurrencia (de una manera cuantitativa y / o cualitativa) 

3. Cómo puede afectar Cómo pueden afectar a la estrategia de la organización y el la estrategia y creación de valor proceso de creación de valor a través del tiempo a través del capital utilizado. (0-2)

4. Fiabilidad e integridad

Fiabilidad e integridad. Incluye los controles que se han aplicado para reducir los errores materiales y un aceptable nivel bajo de riesgo. (Mecanismo, como, por ejemplo, un robusto control interno y sistemas de reporte, los grupos de interés, la auditoría interna o funciones similares, de verificación externa independiente, la limitación de la información, etc.) (0-2)

FACTOR 4. Ambiente externo (Puntaje bruto 0-6)

SUB-FACTOR

1. Ambiente externo de la firma

2. Interacción

\section{EXPLICACIÓN}

Incluye datos significativos sobre el ambiente externo de la empresa, su interacción (con la sociedad en general) y cómo afecta el proceso de creación de valor. (0-2)

En él se describe la interacción, las relaciones y las actividades realizadas por la empresa con el fin de responder a las necesidades de los interesados. Además, cómo esta interacción, las relaciones y las actividades afectan el proceso de creación de valor. (0-2)

3. Estrategia de adaptación y modelo de negocio

Describe cómo la empresa adapta la estrategia y el modelo de negocio para responder al riesgo ambiental externo y oportunidades que enfrentan. (0-2)

FACTOR 5. Gobernabilidad (Puntaje bruto 0-10)

\begin{tabular}{ll}
\hline \multicolumn{1}{c}{ SUB-FACTOR } & \multicolumn{1}{c}{ EXPLICACIÓN } \\
\hline $\begin{array}{l}\text { 1. Pensamiento } \\
\text { integrado }\end{array}$ & $\begin{array}{l}\text { Promueve la toma de decisiones a través de un pensamiento } \\
\text { integrado; se centra en el proceso de creación de valor a través } \\
\text { del tiempo (Concisa, estratégica y con orientación futura). (0-2) }\end{array}$ \\
\hline $\begin{array}{l}\text { Existencia de } \\
\text { una declaración } \\
\text { de los cargos de } \\
\text { gobernabilidad }\end{array}$ & $\begin{array}{l}\text { Existencia de una declaración de los cargos del gobierno, } \\
\text { incluyendo (0-2): }\end{array}$ \\
& a) $\begin{array}{l}\text { Reconocimiento de las responsabilidades garantizando la } \\
\text { integridad de los reportes. }\end{array}$ \\
& b) $\begin{array}{l}\text { Reconocimiento de que el RI se ha preparado en base a } \\
\text { un pensamiento colectivo. }\end{array}$ \\
& c) $\begin{array}{l}\text { Su opinión o declaración de que el RI se realizó de } \\
\text { acuerdo con el IIRF. Si no lo hace de esta forma, se } \\
\text { debería incluir una declaración del gobierno que lo } \\
\text { debiera explicar. } \\
\text { iQué papel jugaron los encargados del gobierno en su } \\
\text { preparación y presentación? }\end{array}$ \\
& d) \\
&
\end{tabular}


3. Políticas $y$ procedimientos
Incluye políticas y procedimientos utilizados en las decisiones estratégicas, apoyando a la capacidad de la empresa para crear valor en el corto, mediano y largo plazo. (0-2)

4. Prácticas del gobierno que exceden los requisitos legales

5. Proceso de remuneración y creación de valor
Describe si la organización está llevando a cabo prácticas de gobierno que exceden los requisitos legales. (0-2)

Describe cómo la remuneración y los incentivos están relacionados con el proceso de creación de valor en el corto, mediano ya largo plazo, y cómo están vinculados con el uso de la organización y los efectos en los capitales elegidos. (0-2)

FACTOR 6. Rendimiento y la perspectiva (Puntaje bruto 0-10)

\begin{tabular}{lll}
\hline \multicolumn{1}{c}{ SUB-FACTOR } & \multicolumn{1}{c}{ EXPLICACIÓN } \\
\hline $\begin{array}{l}\text { 1. } \\
\begin{array}{l}\text { Objetivos } \\
\text { estratégicos a través } \\
\text { del tiempo }\end{array}\end{array}$ & $\begin{array}{l}\text { Describe los objetivos estratégicos a corto, mediano y largo plazo } \\
\text { de la compañía (0-2). }\end{array}$ \\
\hline $\begin{array}{l}\text { 2. } \\
\text { Estrategias en su } \\
\text { sitio }\end{array}$ & $\begin{array}{l}\text { Las estrategias están implementadas, o se tiene la intención } \\
\text { de implementarlas para el logro de los objetivos estratégicos. } \\
\text { Muestran cómo serán medidos (0-2). }\end{array}$ \\
\hline 3. & $\begin{array}{l}\text { Los indicadores } \\
\text { cuantitativos y/o } \\
\text { cualitativos. }\end{array}$ & $\begin{array}{l}\text { Indicadores cuantitativos y/o cualitativos acerca del rendimiento } \\
\text { en el corto, mediano y largo plazo. }\end{array}$ \\
\hline $\begin{array}{l}\text { Los grados que la } \\
\text { compañía logra sus } \\
\text { objetivos }\end{array}$ & $\begin{array}{l}\text { Indica hasta qué punto la empresa ha conseguido sus objetivos } \\
\text { estratégicos. (0-2) }\end{array}$ \\
\hline $\begin{array}{l}\text { Conectividad de } \\
\text { los resultados } \\
\text { financieros } \\
\text { y otros capitales }\end{array}$ & $\begin{array}{l}\text { Medidor de desempeño (Key Performance Indicator - KPI) } \\
\text { demuestran la conectividad de los resultados financieros respecto } \\
\text { a otros capitales. (0-2) }\end{array}$ \\
\hline
\end{tabular}

Tabla 1 muestra el índice de calidad de la divulgación de los RI sobre la base del Marco Conceptual, que comprende seis factores clave: (1) Estructura integrada de informes y comunicación efectiva, (2) Estrategia de organización, (3) Materialidad, (4) Ambiente externo, (5) Gobernabilidad, (6) Rendimiento y su perspectiva. Por cada factor se generan sub-factores. El índex cuenta con un total de 33 sub-factores. 


\section{RESULTADOS}

\section{Características de las organizaciones analizadas}

Del total de compañías analizadas, 20 sociedades anónimas presentaron RI (18,5\% de la muestra inicial). De acuerdo con las actividades económicas que desarrollan un $30 \%$ son productoras y comercializadoras, un $20 \%$ de servicios de transporte, $15 \%$ de servicios financieros y el resto se distribuye en empresas de consumo y servicios.

De la muestra analizada, el 80\% declara preparar los RI bajo los principios establecidos en el Marco Conceptual Internacional de RI. El restante de las empresas no declara e incluso existe reportes que utiliza la metodología del GRI, autodenominando los informes como "Reportes Integrados" o "Memorias Integradas".

\section{Resultado por categoría principales}

Tabla 2 muestra la descripción estadística de las seis categorías. En promedio los elementos que mayor puntaje obtuvieron fueron "Materialidad" con un 61\% y "Estrategia de Organización" con un $60 \%$. Le siguen "Estructura Integrada de Informes y Comunicación Efectiva" y "Ambiente Externo" con un 58\% y 56\% de cumplimiento respectivamente. Resulta llamativo el bajo nivel de cumplimiento para los elementos de "Rendimiento y Perspectivas" y "Gobernabilidad" considerando que ambos criterios se pueden encontrar a modo de resumen al principio de los informes que explican los mecanismos de gobernanza vigentes. Si se analiza el índice en su totalidad, el mejor RI divulgado obtuvo un puntaje de 65\% mientras que el puntaje menor fue de 33\% con un promedio del $51 \%$ lo que demuestra la discrecionalidad de las divulgaciones presentadas.

\section{Tabla 2}

\section{Descripción estadística.}

\begin{tabular}{lccccc}
\hline Categorías & Mínimo & Máximo & Media & Mediana & $\begin{array}{c}\text { Desv. } \\
\text { Estand }\end{array}$ \\
\hline $\begin{array}{l}\text { Estructura integrada de informes } \\
\text { y comunicación efectiva }\end{array}$ & 0,17 & 0,75 & 0,58 & 0,63 & 0,16 \\
\hline Estrategia de organización & 0,35 & 0,80 & 0,60 & 0,60 & 0,14 \\
\hline Materialidad & 0,00 & 0,88 & 0,61 & 0,63 & 0,26 \\
\hline Ambiente externo & 0,17 & 1,00 & 0,56 & 0,50 & 0,24 \\
\hline Gobernabilidad & 0,00 & 0,60 & 0,28 & 0,30 & 0,17 \\
\hline Rendimiento y la perspectiva & 0,10 & 0,60 & 0,40 & 0,50 & 0,15 \\
\hline Total Índice & 0,33 & 0,65 & 0,51 & 0,52 & 0,10 \\
\hline
\end{tabular}

Tabla 2 muestra la descripción estadística de los seis factores principales (o categorías) del índice (detallado en Tabla 1), y evaluados de manera independiente. La última línea, denominada Total índice, muestra la evaluación total de los reportes analizados, el cual se obtiene dividiendo el total de puntos obtenidos en todas las categorías, por el máximo de puntos del índex (66 puntos). 


\section{Resultados por subcategorías}

Los resultados por subcategorías son presentados en Tabla 3 panel A y B.

\section{Factor 1. Estructura integrada de informes y comunicación efectiva}

El Factor 1, el cual evalúa la estructura integrada de los informes y su comunicación efectiva se evalúa en 6 sub-factores y es presentado en Tabla 3, Panel A, Columna A. La mayoría de los RI analizados $(80 \%)$ se preparan en respuesta a lo establecido por el International Integrated Reporting Framework tomando como referencia y base de preparación el Marco Conceptual publicado por The International Integrated Reporting Council (IIRC). De la misma manera existe una adecuada legibilidad a través del uso de referencias dentro de documentos cruzadas limitando la repetición y mejorando la lectura del informe.

Por otro lado, no se aprecia un entendimiento claro en las empresas que divulgan RI en relación con la conectividad de la información y la interdependencia sobre los factores que afectan la capacidad de crear valor. En este caso, la conectividad de la información debe estar ligada no sólo a cómo los capitales de la compañía generar valor en el corto, mediano y largo plazo, sino también una interconexión entre la información financiera y no financiera, los gobiernos corporativos y la estrategia organizacional. Se puede observar además que sólo el 5\% de las empresas incluye una descripción clara de la dimensión del tiempo futuro (corto, mediano y largo plazo) para la preparación y presentación de los RI.

\section{Factor 2. Estrategia de organización}

La subclasificación Estrategia de la Organización, con un promedio de cumplimiento de un $59,5 \%$, se muestra en Tabla 3, Panel A, Columna B. A pesar de que, en términos generales, presenta niveles de cumplimiento superiores a su media en aspectos como divulgación de la misión y visión empresarial, actividades comerciales, riesgos y oportunidades, cultura, entre otros; estos aspectos son comúnmente divulgados al momento de proveer información corporativa, independientemente de si se realiza a través de un informe integrado o un reporte de sustentabilidad. Lo que llama la atención de esta subcategoría, es la poca comprensión por parte de las empresas en la descripción de los recursos (capitales) utilizados para la creación de valor. En teoría, el principal enfoque de los RIs debería ser el efecto de los capitales utilizados en el proceso de creación de valor. Los capitales, no son completamente independientes; su interacción depende de diferentes factores como el tipo de industria, modelo de negocio y otros asuntos externos e internos que afectan a la organización. Por lo tanto, el propósito de categorizar el capital en la creación de valor es asegurar que las empresas consideren todo tipo de capital que pueda afectar materialmente el valor creado. El modelo de negocio juega un papel principal en aclarar cómo interactúan las diferentes actividades para crear valor a lo largo del tiempo. Al estudiar estos factores en las empresas analizadas, denota falta de comprensión por parte de las organizaciones al momento de preparara y divulgar los RI. 


\section{Factor 3. La materialidad}

Factor materialidad se evalúa a través de cuatro sub-factores: (1) La existencia de un proceso; (2) La forma en que se han determinado y cuantificado; (3) Cómo puede afectar la estrategia y creación de valor; y (4) La fiabilidad e integridad. El análisis se muestra en Tabla 3, Panel A, Columna C. En su mayoría las empresas divulgan la existencia de un proceso que determina los aspectos materiales y la forma en que lo han cuantificado (sobre un $80 \%$ de cumplimiento). En un menor grado ( $55 \%$ de cumplimiento) las empresas divulgan cuáles son los controles que se han aplicado para reducir los errores materiales con el objetivo de aumentar la fiabilidad e integridad. Por el contrario, los resultados muestran que las empresas no logran divulgar y relacionar en su mayoría el cómo la materialidad puede afectar a la estrategia y el proceso de creación de valor a través del tiempo. Esto es un resultado esperado considerando que, en términos generales, los reportes carecen de información respecto a la comprensión e interdependencia de los capitales utilizados en el período evaluado (subcategoría 2).

\section{Factor 4. Ambiente externo}

Los factores relacionados con el ambiente externo se muestran en Tabla 3, Panel B, Columna D. Este factor se evalúa en tres ámbitos: (1) ambiente externo de la firma, (2) la interacción de la empresa con el fin de responder a las necesidades de las partes interesadas y su relación con la creación de valor, y (3) la estrategia de adaptación y modelo de negocios para responder al riesgo ambiental y oportunidades. En un 65\% las empresas divulgan datos significativos sobre el ambiente externo, principalmente su interacción con la sociedad. En un mismo porcentaje, las empresas describen cómo adaptan las estrategias y el modelo de negocio para responder a los riegos ambientales y las oportunidades que les ofrece el ambiente externo. Sin embargo, no se explica claramente (un 37,5\%) su interacción en el proceso de creación de valor. Es decir no existe claridad al momento de describir la interacción, las relaciones y las actividades realizadas, con el fin de responder a las necesidades de los stakeholder y cómo toda esa interacción afecta a la creación de valor.

\section{Factor 5. Gobernabilidad}

Gobernabilidad se analiza a través de cinco factores (1) Pensamiento integrado; (2) Declaración cargos de gobernabilidad; (3) Políticas y procedimientos; (4) Prácticas que exceden los requisitos legales; y (5) proceso de remuneración y creación de valor (ver Tabla 3, Panel B, Columna E). Los resultados sugieren que las empresas promueven la toma de decisiones a través de un pensamiento integrado, centrado en proceso en la creación de valor y orientación futura (50\% de cumplimiento). En un menor porcentaje (45\%), declara los cargos de gobierno corporativo en base al reconocimiento de las responsabilidades sobre la integridad de la información presentada y su pensamiento colectivo en base a lo establecido en el Marco Conceptual. Por su parte, los resultados muestran un $21 \%$ de cumplimiento promedio en la divulgación de políticas y procedimientos en las decisiones estratégicas, apoyando a la capacidad de la empresa a crear valor. En un mismo porcentaje, las empresas describen si se está está llevando a cabo prácticas de gobierno que exceden los requisitos legales. Similarmente, y sólo con un 2,5\% de cumplimiento, las organizaciones describen cómo los incentivos están relacionados a la creación de valor en el tiempo y su efecto en los capitales elegidos. 


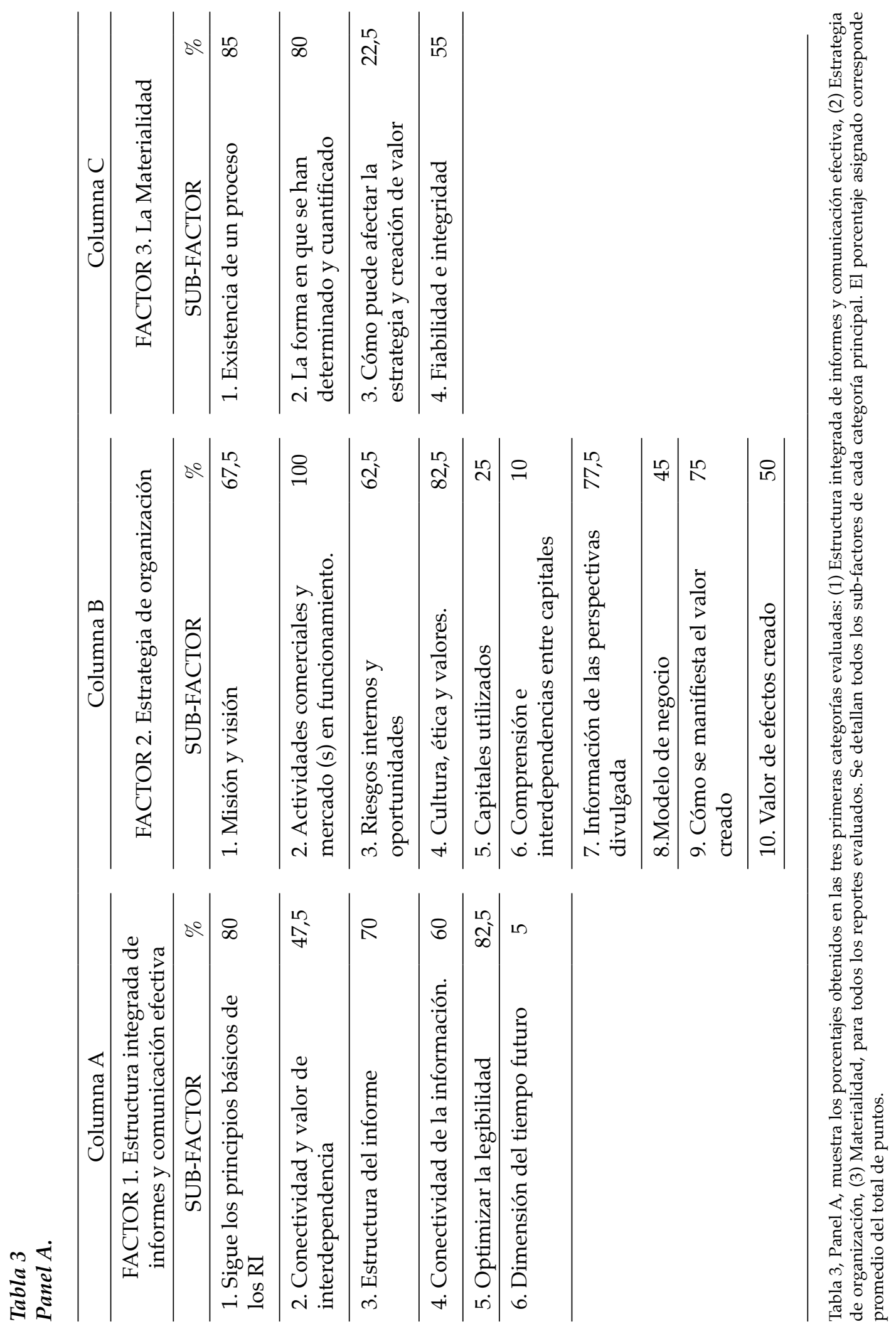




\section{Factor 6. Rendimiento y perspectiva}

Rendimiento y perspectiva, presentado en Tabla 3, Panel b, Columna F, se analiza en 5 aspectos: (1) Objetivos estratégicos a través del tiempo; (2) Estrategias en su sitio; (3) Los indicadores cuantitativos y/o cualitativos: (4) Los grados que la compañía logra sus objetivos y (5) Conectividad de los resultados financieros y otros capitales. Los resultados muestran que en un gran porcentaje $(80 \%)$ las organizaciones muestran indicadores cuantitativos y/o cualitativas acerca del rendimiento en el corto, mediano y largo plazo.

En un porcentaje un poco menor $(63 \%)$, los reportes analizados indican cuáles han sido las estrategias implementadas para el logro de los objetivos estratégicos y cómo son o serán medidos. Contrario a las expectativas, y en un porcentaje menor (43\%) se describen los objetivos estratégicos en el tiempo. Con un $13 \%$ se indica los grados que la compañía logra sus objetivos y la conectividad de los resultados financieros y otros capitales. Finalmente, y contrario a las expectativas, ninguna compañía (0\%) divulga medidores de desempeño (KPI - Key Performance Indicator), que conecten los resultados financieros con los otros capitales establecidos por la compañía.

\section{DISCUSION Y CONCLUSIONES}

Se ha argumentado que la información financiera divulgada por las empresas a través de las políticas y reglas contables existentes no refleja completamente cómo los factores no financieros pueden afectar el desempeño actual y futuro de una empresa, dificultando la evaluación de los gobiernos corporativos y desempeño. En consecuencia, el IIRC publica el Marco Conceptual para la preparación y presentación de RI, con el objetivo de integrar la información financiera y no financiera en un solo informe. Para ello se desarrolló un modelo coherente capaz de proporcionar un conjunto completo de información de la empresa basado en los objetivos estratégicos, modelo de gobierno corporativo y estrategia de negocio (IIRC, 2013c). El principal enfoque de los RI es mostrar cómo las empresas utilizan los capitales identificados en el proceso de creación de valor a lo largo del tiempo.

Motivado por la necesidad de comprender qué se está divulgando como información integrada y cuáles son factores asociados a un reporte de alta calidad, se denota que los reportes tienden a mostrar una comunicación efectiva; sin embargo, hay aspectos que requieren de mayor atención. Por ejemplo, existe poca claridad al momento de proporcionar conectividad e interdependencia de los factores que afectan la capacidad de crear valor. Del mismo modo, si bien existe un proceso que determina los aspectos materiales, cómo se han determinado y, existe un poco integración en cómo puede afectar la materialidad en la estrategia de la empresa y en la creación de valor.

En términos generales se concluye que la divulgación de RIs en el mercado chileno se encuentra en una etapa muy temprana, inmadura y con un alto desconocimiento en el foco y el objetivo de presentar la información integrada. Si bien hay un esfuerzo en ir avanzando en materias de gobiernos corporativos, existe un alto grado de discrecionalidad en la información presentada que tiende a mitigar los potenciales beneficios de estos reportes. 


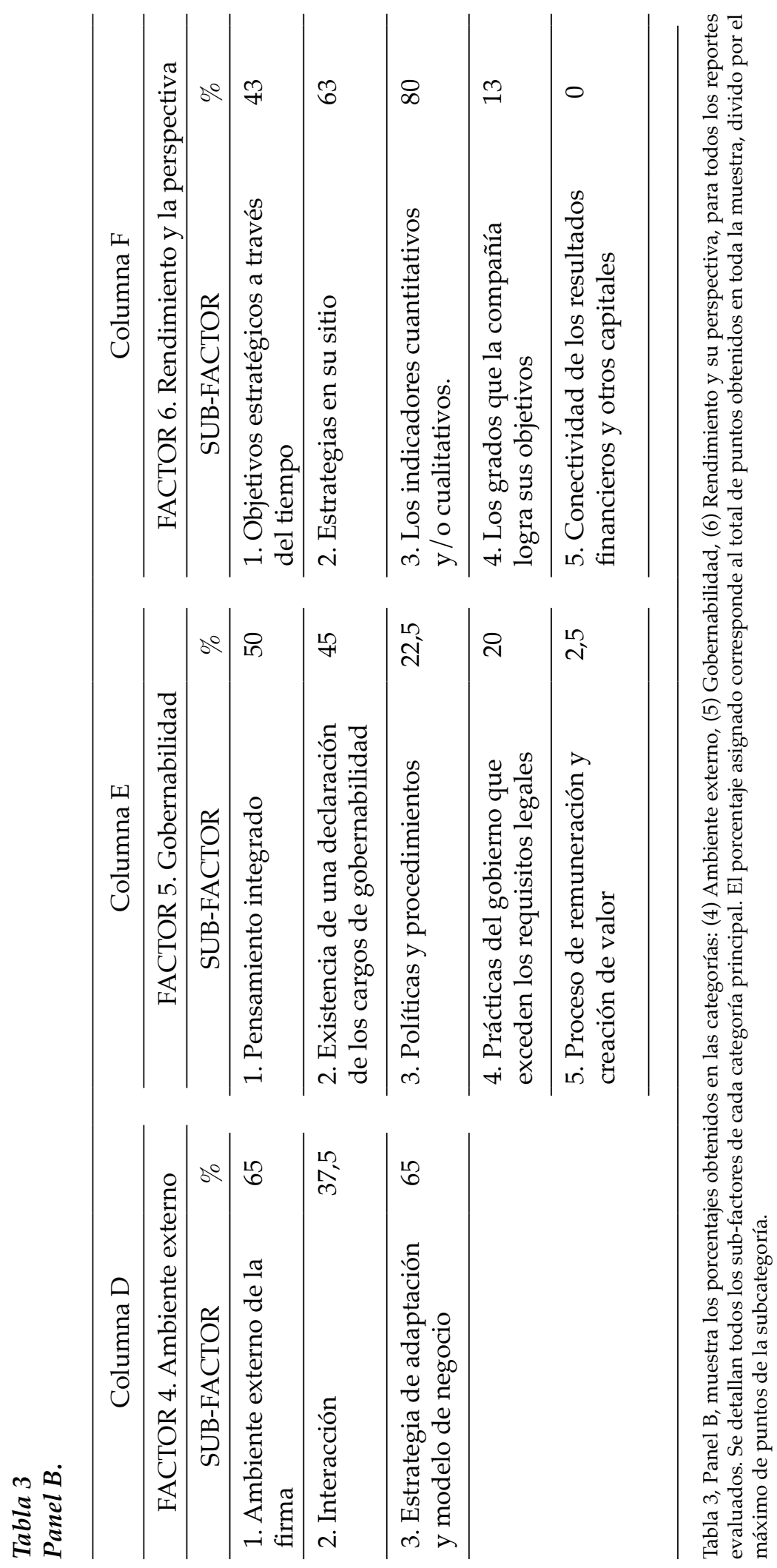


Se debe tener precaución al evaluar la información corporativa a través de RI cuando no existe un análisis profundo del espíritu de entregar la información integrada de acuerdo con el Marco Conceptual. Integrar la información no es sólo juntar los reportes financieros y de sustentabilidad en un solo reporte y llamarlo integrado, es un análisis hacia los proveedores de capital explicando cómo la compañía, a través de todos sus recursos (capitales) crean valor. Es aquí donde se encuentra la principal deficiencia; cómo identificar y reportar los capitales y, por lo tanto, cómo su interacción crea valor. Las divulgaciones sobre los capitales utilizados se determinan de acuerdo con la capacidad de una empresa para crear valor a corto, mediano y largo plazo, más que si son propiedad de la organización o no. Es más, el Marco Conceptual proporciona una guía teórica para que las empresas se aseguren de que se divulguen todas las formas de capital. Si bien el IIRC respalda las seis formas de capital (financiero, manufacturado, intelectual, humano, social y relacional, y natural), no requiere que las empresas lo adopten de la misma forma. En cambio, las empresas pueden tomar diferentes opciones para informar mejor las interconexiones entre las formas de capital. El pilar de los RI es la mejora continua en la presentación de la información corporativa (accountability) y la administración de los capitales, promoviendo la interdependencia y el entendimiento entre ellos.

En esta investigación, la descripción estadística muestra que el informe con mayor puntaje obtuvo un 65,2\% de cumplimiento, el mínimo 33\% y una media de 51\%, lo que muestra la discrecionalidad de la información divulgada. Por un lado, esto podría sugerir, por ejemplo, desconocimiento al momento de preparar la información integrada; por otro lado, podría representar cierta mímica de las organizaciones a través del envío de señales equivocadas al mercado. Si bien existe evidencia de los beneficios de los RI, estos se encuentran asociados a la calidad de la información presentada (Barth et al., 2017; Zhou et al., 2017; Zúñiga, Pincheira, Walker, et al., 2020). La administración, a través de la teoría de la agencia, tiene incentivos para buscar una mayor credibilidad de la información reportada como un medio de enviar señales al mercado mostrando una mayor capacidad de administración (Verrecchia, 1990). La calidad de la información y la reputación corporativa pueden usarse como una señal para los accionistas para diferenciar la "buena ciudadanía corporativa" de las empresas "greenwash". Sin embargo, las señales engañosas enviadas al mercado a través de la divulgación de información incompleta e inexacta pueden afectar negativamente las percepciones de las partes interesadas. Un análisis de contenido exhaustivo y sus efectos en los mercados de capitales podría ayudar a aclarar si la divulgación de los RI se ha utilizado como una señal para el mercado o si es parte de un comportamiento de imitación para las empresas que no pueden divulgar información de alta calidad. ¿Existe un esfuerzo genuino de divulgar voluntariamente información integrada? $\mathrm{O}$ sólo corresponde a un "marketing inteligente". Aún existen muchas interrogantes que podrían ser dilucidadas en investigaciones futuras. 


\section{REFERENCIAS}

Barth, M., Cahan, S., Chen, L., y Venter, E. (2017). The Economic consequences associated with integrated report quality: Capital market and real effects. Accounting, Organizations and Society, 62, 43-64.

Bernardi, C., y Stark, A. W. (2016). Environmental, social and governance disclosure, integrated reporting, and the accuracy of analyst forecasts. The British Accounting Review.

Brown, J., y Dillard, J. (2014). Integrated reporting: On the need for broadening out and opening up. Accounting, Auditing \& Accountability Journal, 27(7), 1120-1156.

Dhaliwal, D. S., Li, O. Z., Tsang, A., y Yang, Y. G. (2011). Voluntary nonfinancial disclosure and the cost of equity capital: The initiation of corporate social responsibility reporting. The Accounting Review, 86(1), 59-100.

Dhaliwal, D. S., Radhakrishnan, S., Tsang, A., y Yang, Y. G. (2012). Nonfinancial disclosure and analyst forecast accuracy: International evidence on corporate social responsibility disclosure. The Accounting Review, 87(3), 723-759.

Diamond, D. W., y Verrecchia, R. E. (1991). Disclosure, liquidity, and the cost of capital. The Journal of Finance, 46(4), 1325-1359.

Guidry, R., y Patten, D. (2010). Market reactions to the first-time issuance of corporate sustainability reports. Evidence that quality matters. Sustainability Accounting, Management and Policy Journal, 1(1), 33-50.

Herbohn, K., Walker, J., y Loo, H. Y. M. (2014). Corporate social responsibility: The link between sustainability disclosure and sustainability performance. Abacus, 50(4), 422-459.

Higgins, C., Stubbs, W., y Love, T. (2014). Walking the talk(s): Organisational narratives of integrated reporting. Accounting, Auditing \& Accountability Journal, 27(7), 1090-1119.

IIRC. (2013a). Capitals background paper for IR: International Integrated Reporting Council.

IIRC. (2013b). The International <IR $>$ Framework: International Integrated Reporting Council.

IIRC. (2013c). The International $<\mathrm{IR}>$ Framework Released with Business and Investor Support. Retrieved from http://www.theiirc.org/2013/12/09/the-international-ir-framework-released-with-business-and-investor-support /

IIRC. (2015). Creating value. Integrated reporting $<\mathrm{IR}>$ and investor benefits: International Integrated Reporting Council.

Lu, C.-W., y Chueh, T.-S. (2015). Corporate Social Responsibility and Information Asymmetry. Journal of Applied Finance and Banking, 5(3), 105-122.

Ocean Tomo. (2015). Intellectual capital equity. Retrieved from http://www.oceantomo.com/ intellectual-capital-equity /

Qiu, Y., Shaukat, A., y Tharyan, R. (2016). Environmental and social disclosures: Link with corporate financial performance. The British Accounting Review(0). doi:https:/ / doi.org/10.1016/j. bar.2014.10.007

Stent, W., y Dowler, T. (2015). Early assessments of the gap between integrated reporting and current corporate reporting. Meditari Accountancy Research, 23(1), 92.

Stubbs, W., y Higgins, C. (2014). Integrated reporting and internal mechanisms of change. Accounting, Auditing \& Accountability Journal, 27(7), 1068-1089.

Norma de caracter general 385, (2015).

van Bommel, K. (2014). Towards a legitimate compromise? : An exploration of Integrated Reporting in the Netherlands. Accounting, Auditing \& Accountability Journal, 27(7), 1157-1189. 
Verrecchia, R. E. (1990). Information quality and discretionary disclosure. Journal of Accounting and Economics, 12(4), 365-380.

Zhou, S., Simnett, R., y Green, W. (2017). Does integrated reporting matter to the capital market? Abacus, 53(1), 94-132.

Zúñiga, F., Pincheira, R., Aguilar, C., y Silva, J. (2020). Informes de sustentabilidad y su auditoría: Efecto en la liquidez de mercado chileno. Estudios Gerenciales, 36(154), 56-65. doi:https:/ / doi.org/10.18046/j.estger.2020.154.3558

Zúñiga, F., Pincheira, R., Dimter, M., y Quinchel, B. (2021). Reportes integrados y la auditoría nofinanciera: Efectos económicos. Working paper. SSRN. Retrieved from http://ssrn.com/abstract $=3803195$.

Zúñiga, F., Pincheira, R., Walker, J., y Turner, M. (2020). The effect of integrated reporting quality on market liquidity and analyst forecast errors. Accounting Research Journal, 33(4/5), 635650.

\section{(c) $(1)(9)$}

Esta obra está bajo una licencia de Creative Commons Atribución-NoComercial-CompartirIgual 4.0 Internacional 